\title{
Influence of physical and geometrical parameters on electrical properties of short gate GaAs MESFETs
}

\author{
S. Khemissi ${ }^{2}$, N. Merabtine ${ }^{1}$, M. Zaabat ${ }^{3}$, C. Kenzai ${ }^{4}$, Y. Saidi ${ }^{4}$, S. Amourache ${ }^{4}$ \\ ${ }^{1}$ Laboratoire Electromagnetisme et Telecommunication, Electronics department, \\ Faculty of Engineering, University Mentouri Constantine, Algeria \\ E-mail:na_merabtine@hotmail.com. \\ ${ }^{2}$ Departement de Seti, Faculté de technologie, Université de khenchla, Algeria \\ E-mail: saadekhemissi@yahoo.fr \\ ${ }^{3}$ Physics department, University of Oum-El-Bouaghi, Algeria \\ E-mail: Zaabat@hotmail.com. \\ ${ }^{4}$ Laboratoire des couches minces et interfaces, Département de physique, Faculté des Sciences, \\ Université Mentouri de Constantine, Algeria \\ E-mail: musbelgat@yahoo.fr
}

\begin{abstract}
In the information sciences such as computer science, telecommunications, the treatment of signal or image transmission, the field effect components play an important role. In the frame of our work, we are interested in the study of the gallium arsenide short gate field effect transistor called GaAs MESFET. After analytical studying the component static characteristics, according to different operation regimes, a numerical simulation was worked out. The influence of technological dimensions $\left(L, Z, a\right.$, and $\left.N_{d}\right)$ was studied. The obtained results allow us to determine optimal parameters of the devices from the viewpoint of their applications and specific use.
\end{abstract}

Keywords: GaAs MESFET, technological, physical and geometrical parameters, parasite elements.

Manuscript received 09.01.06; accepted for publication 29.03.06.

\section{Introduction}

Several research laboratories showed the interest of using the Gallium Arsenide Field Effect Transistor (GaAs MESFET) for the realization of analog and logic integrated circuits. A fairly good technological realization can only be achieved with a deep knowledge of the component physics and all the intrinsic and extrinsic phenomena that can limit its performances. In this paper, we propose an analytical model (simulation) of current-voltage characteristics of a short gate GaAs MESFET, then we have elaborated a software that enable us to solve the system of differential equations and plot a series of different curves.

\section{Determination of the voltage under the gate}

The two dimensional solution of differential equations using the Green method gives a distribution of the electric field under the region of the space charge area (SCA).
To calculate the voltage under the gate, the SCA is divided in two main regions [1].

- The region (1) directly under the gate, considered as a region controlled by the gate. We use the uni-dimensional approximation to calculate the component of the expression for the voltage $V_{q}(x, y)$ specific to this region.

- The region (2) outside the first region considered to be uncontrolled by the gate. The twodimensional voltage of the channel under the gate is given as follows:

$V_{c}(x, y)=V_{q}(x, y)+V_{l}(x, y)$.

Where

$$
\begin{aligned}
& V_{q}(x, y)=\int_{0}^{y} \frac{e N_{d}(x, y)}{\varepsilon} y d y+ \\
& +y \int_{y}^{h(x)} \frac{e N_{d}(x, y)}{\varepsilon} d y+V_{b i}-V_{g}
\end{aligned}
$$


and

$$
\begin{aligned}
& V_{l}(x, y)=\left[A_{1}^{s} \frac{\sinh \left(k_{1}(L-x)\right)}{\sinh \left(k_{1} L\right)}+\right. \\
& \left.+A_{1}^{d} \frac{\sinh \left(k_{1} x\right)}{\sinh \left(k_{1} L\right)}\right] \sin \left(k_{1} y\right)
\end{aligned}
$$

with

$$
A_{1}^{S}=\frac{2}{a} \int_{0}^{a}\left[V_{c}(0, y)-V_{q}(0, y)\right] \sin \left(k_{1} y\right) d y
$$

and

$$
A_{1}^{d}=\frac{2}{a} \int_{0}^{a}\left[V_{c}(L, y)-V_{q}(L, y)\right] \sin \left(k_{1} y\right) d y
$$

$A_{1}^{d}$ and $A_{1}^{S}$ are the Fourier coefficients for the gate supplementary for voltage drain and source sides, respectively, [1]

and $k_{1}=\frac{\pi}{2 a}$.

From (3) and (4) the total voltage expression is written as:

$V_{c}(x, y)=\int_{0}^{h(x)} \frac{e N_{d}(x, y)}{\varepsilon} y d y+V_{l}(x, y)-V_{g}+V_{b i}$.

\section{Effect of the mobility law}

The constant mobility hypothesis and independent of the electric field in the $n$-type GaAs cannot convey the physical phenomena. The analytical expression of the variations of the mobility with the electric field which we use is a simplified relation [2-4] given as follows:

- for the weak electric field where $E<E_{0}$

$\mu=\mu_{0}$,

- for the high electric field beyond $E_{0}\left(E>E_{0}\right)$

$$
\mu=\frac{\mu_{0}}{\left[1+\left(\frac{E-E_{0}}{E_{S}}\right)^{2}\right]^{1 / 2}} .
$$

This mobility law allows us to obtain the different expressions of the drain current in different operation regimes.

\section{Current-voltage characteristics}

To calculate the drain current expression as a function of the drain voltage for different values of the gate voltage, we use the following hypothesis:
- we neglect the current along the $Y$ axis [5], this approximation is valid for the short gate components;

- we suppose the electron mobility to be variable according to the zones under the gate;

- we derived the channel in three regions according to the electric field value (Fig. 1) $[6,7]$.

The $I_{d}\left(V_{d}, V_{g}\right)$ characteristics of the GaAs MESFET transistor corresponding to different operation regimes are ruled by the following equations.

\subsection{Linear regime}

This regime exists as far as $L_{a}$ occupies whole channel, it corresponds to weak field sphere where the mobility is equal to $\mu_{0}$.

The drain current expression in this regime is given as:

$I_{d}=I_{p l}\left[\frac{V_{d}}{V_{p}}-\frac{2}{3}\left(\frac{2}{3} \frac{V_{d}+V_{b i}-V_{g}}{V_{p}}\right)^{3 / 2}+\right.$

$\left.+\frac{2}{3}\left(\frac{V_{b i}-V_{g}}{V_{p}}\right)^{3 / 2}\right]$

where

$I_{p l}=\frac{e^{2} N_{d}^{2} Z \mu_{0} a^{3}}{2 \varepsilon L_{a}}$.

\subsection{Pinch-off regime}

As the drain voltage increases, the electric field in the channel increases beyond $E_{0}$. Then the channel under the gate presents two regions.

One region with the length $L_{a}$ in which the field is inferior to $E_{0}$ and the electron mobility is constant and given by $\mu=\mu_{0}$. The other region with the length $L_{b}(L=$ $\left.=L_{a}+L_{b}\right)$ in which the field is superior to the field $E_{0}$, but inferior to the field $E_{m}$ and the electron mobility is given by the expression (7b).

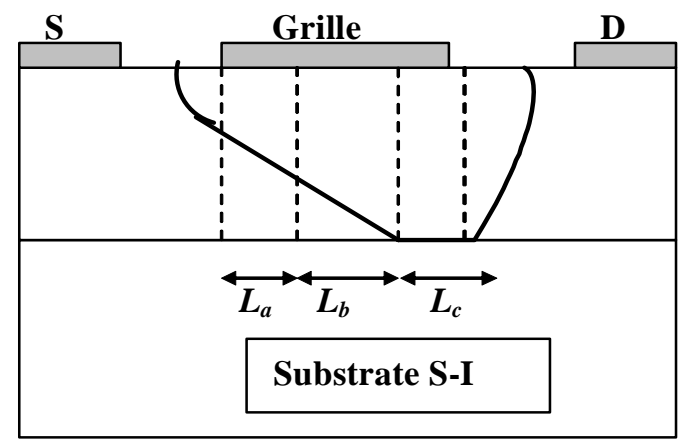

Fig. 1. Active zone repartions according to the electrical field variation. 
First region: for $E<E_{0}$ and $0<x<L_{a}$

$L_{a}=\frac{I_{p l} L}{I_{d}}\left[\frac{V_{d a}}{V_{p}}-\frac{2}{3}\left(\frac{V_{d_{a}}+V_{b i}-V_{g}}{V_{p}}\right)^{3 / 2}-\frac{2}{3}\left(\frac{V_{b i}-V_{g}}{V_{p}}\right)^{3 / 2}\right]$

Second region: for: $E_{0}<E<E_{m}$ and $L_{a}<x<L$

$$
\begin{aligned}
& L_{b}=\frac{I_{p s} L}{I_{d}}\left[\frac{V_{d}-V_{d a}}{V_{p}}-\frac{2}{3}\left(\frac{V_{d}+V_{b i}-V_{g}}{V_{p}}\right)^{3 / 2}+\right. \\
& \left.+\left(\frac{V_{d a}+V_{b i}-V_{g}}{V_{p}}\right)^{3 / 2}\right],
\end{aligned}
$$

where

$$
I_{p s}=\frac{I_{p}}{\left[1+\left(\frac{E-E_{0}}{E_{s}}\right)^{2}\right]^{1 / 2}} .
$$

\subsection{Saturation regime}

In this case, the channel under the gate is divided into three regions $L_{a}, L_{b}$ and $L_{c}$ where $L=L_{a}+L_{b}+L_{c}$,

$$
\begin{aligned}
& L_{a}=\frac{I_{p l} L}{I_{d}}\left[\frac{V_{d a}}{V_{p}}-\frac{2}{3}\left(\frac{V_{d a}+V_{b i}-V_{g}}{V_{p}}\right)^{3 / 2}-\frac{2}{3}\left(\frac{V_{b i}-V_{g}}{V_{p}}\right)^{3 / 2}\right], \\
& L_{b}=\frac{I_{p s} L}{I_{d}}\left[\frac{V_{d m}-V_{d a}}{V_{p}}-\right. \\
& \left.-\frac{2}{3}\left(\frac{V_{d m}+V_{b i}-V_{g}}{V_{p}}\right)^{3 / 2}+\frac{2}{3}\left(\frac{V_{d a}+V_{b i}-V_{g}}{V_{p}}\right)^{3 / 2}\right], \\
& L_{c}=\frac{I_{p s} L}{I_{d}}\left[\frac{V_{d}-V_{d m}}{V_{p}}-\frac{2}{3}\left(\frac{V_{d}+V_{b i}-V_{g}}{V_{p}}\right)^{3 / 2}+\right. \\
& \left.+\frac{2}{3}\left(\frac{V_{d m}+V_{b i}-V_{g}}{V_{p}}\right)^{3 / 2}\right],
\end{aligned}
$$

where $V_{d a}$ and $V_{d m}$ are successively maxima and pinchoff voltages for linear regimes.

\section{Effect of parasite elements}

The characteristics that we have presented are those concerning internal or intrinsic dimensions $\left(I_{d}, V_{d}, V_{g}\right)$. To obtain the external or extrinsic characteristics $\left(I_{d s}\right.$, $\left.V_{d s}, V_{g s}\right)$ of the component we have to take into consideration the effect of the parasite access source resistance $R_{s}$, the drain resistance $R_{d}$ as well as the effect of the resistance $R_{p}$ parallel to the channel on the polarization voltages values.

To obtain the real expressions of the characteristics $I_{d s}\left(V_{d s}, V_{g s}\right)$, we have to substitute the intrinsic terms by the extrinsic terms in all the previous relations.

Therefore:

$V_{d}=V_{d s}+V_{l d}-\left(R_{s}+R_{d}\right) I_{d}$

$V_{g}=V_{g s}+V_{l s}-R_{s} I_{d}$

$I_{d}=I_{d s}-\left(V_{d} / R_{p}\right)$.

\section{Results and discussion}

In order to validate the $I-V$ characteristics of the GaAs MESFET set up in the previous work, a simulation software based on different formulas and equations is used as well as the obtained results and their discussions.

\subsection{Simulation software}

The simulation software is realized with FORTRAN 32, version 01, from the expressions obtained in the previous paragraphs. It allows the resolution of equation systems and the edition of the results in specific files [8].

With this software we are able to determine:

- the current-voltage characteristics in different operation regimes;

- the effect of parasite resistances on the currentvoltage characteristics;

- the effect of $V_{l s}$ and $V_{l d}$ voltages on the currentvoltage characteristics;

- the effect of geometrical and technological parameters $\left(L, a, Z\right.$, and $\left.N_{d}\right)$ on the current-voltage characteristics.

\subsection{Current-voltage characteristics}

The numerical calculations (Fig. 2) of the drain current as a function of the polarization voltage was carried out by the expressions (8)-(13) previously derived.

The study was carried out on two transistors MESFET 1 and MESFET 2, parameters of which are gathered in Table 1.

Table 1.

\begin{tabular}{|c|c|c|c|c|c|c|c|c|}
\hline Transistor & $L(\mu \mathrm{m})$ & $A(\mu \mathrm{m})$ & $Z(\mu \mathrm{m})$ & $\mu_{0}\left(\mathrm{~m}^{2} / \mathrm{Vcm}\right)$ & $N_{d}\left(10^{23} / \mathrm{m}\right)$ & $V_{s}(\mathrm{~m} / \mathrm{s})$ & $V_{b i}(\mathrm{~V})$ & $V_{p}(\mathrm{~V})$ \\
\hline MESFET 1 & 1 & 0.153 & 300 & 0.4000 & 1.17 & $3.610^{3}$ & 0.85 & 1.93 \\
\hline MESFET 2 & 0.5 & 0.1435 & 300 & 0.4000 & 1.31 & $7.310^{3}$ & 0.85 & 1.93 \\
\hline
\end{tabular}




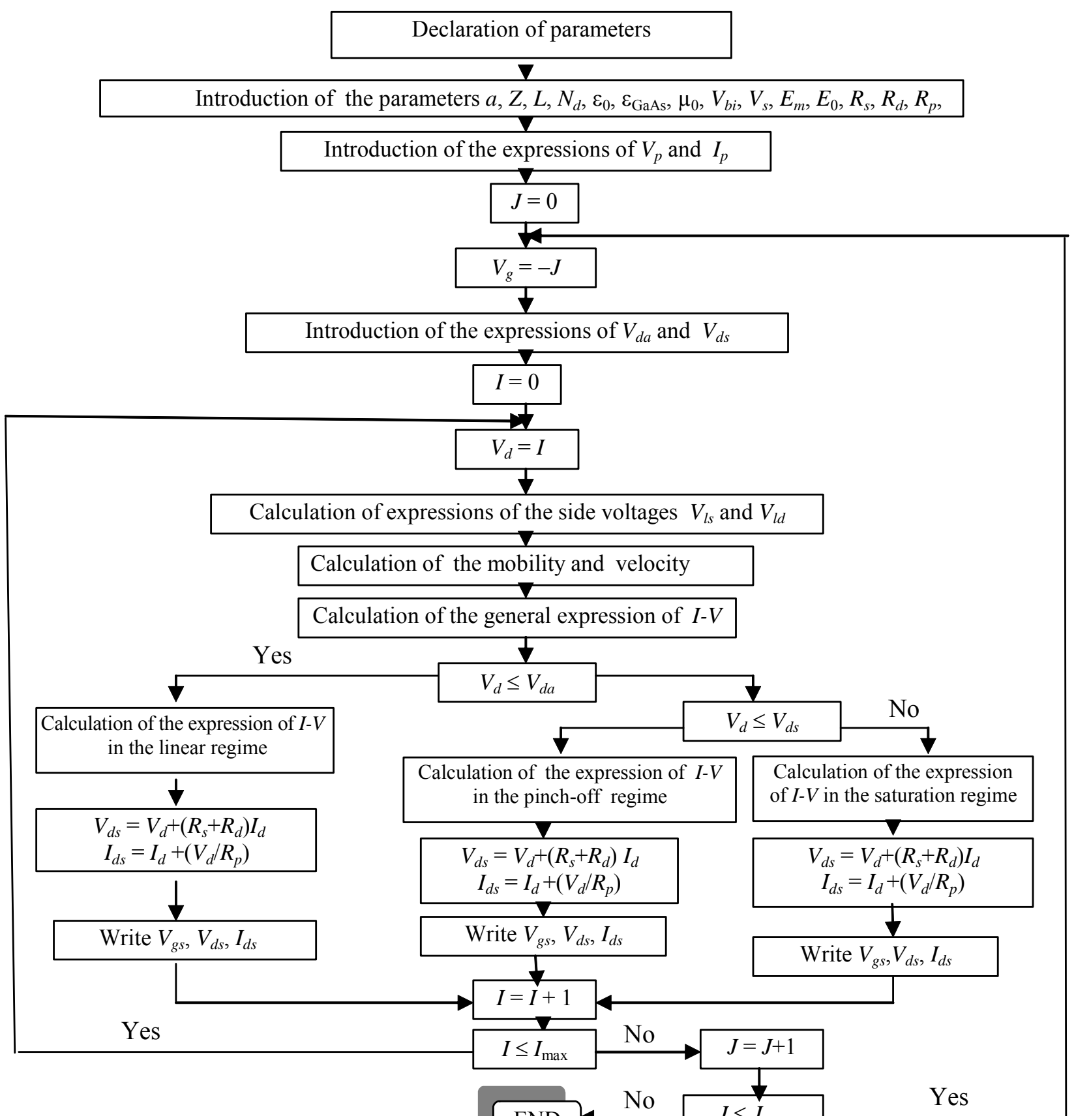

In Figs $3 \mathrm{a}$ and $\mathrm{b}$, we have compared the measured $I_{d s}\left(V_{d s}, V_{g s}\right)$ characteristics and the calculated ones by the simulation for the transistors MESFET 1 and MESFET 2. In the linear regime, i.e., at weak drain voltage polarization, we notice a good agreement between the experimental values and the simulation ones for both transistors. When the drain voltage increases and becomes more important, we notice a certain gap between the experimental values and the results of the simulation. This gap progressively increases until the saturation. This gap is mainly caused by the approximations made in the mathematical model and simulation software; it is also due to the geometric parameter effects and the existence of parasitic quantum phenomena that we have not taken into consideration. In the saturation regime, when the drain voltage gets important, we notice that the theoretical results are in good agreement with the experimental ones. In conclusion, we also remark that the theoretical and experimental results have the same behavior towards the drain voltage and coincide well, notably at high values of the $V_{d s}$ voltage. This shows that the method is well founded.

\subsection{Effect of source and drain parasite resistances}

In order to put into evidence the effects of the source and drain parasite resistances $R_{s}$ and $R_{d}$ on the characteristics $I-V$ of the GaAs MESFET, which we present in Figs 4a and $b$, in the case of the previous two transistors, the variations of the drain current as a function of the drain voltage with and without the parasite resistance were estimated.

\section{(C) 2006, V. Lashkaryov Institute of Semiconductor Physics, National Academy of Sciences of Ukraine}



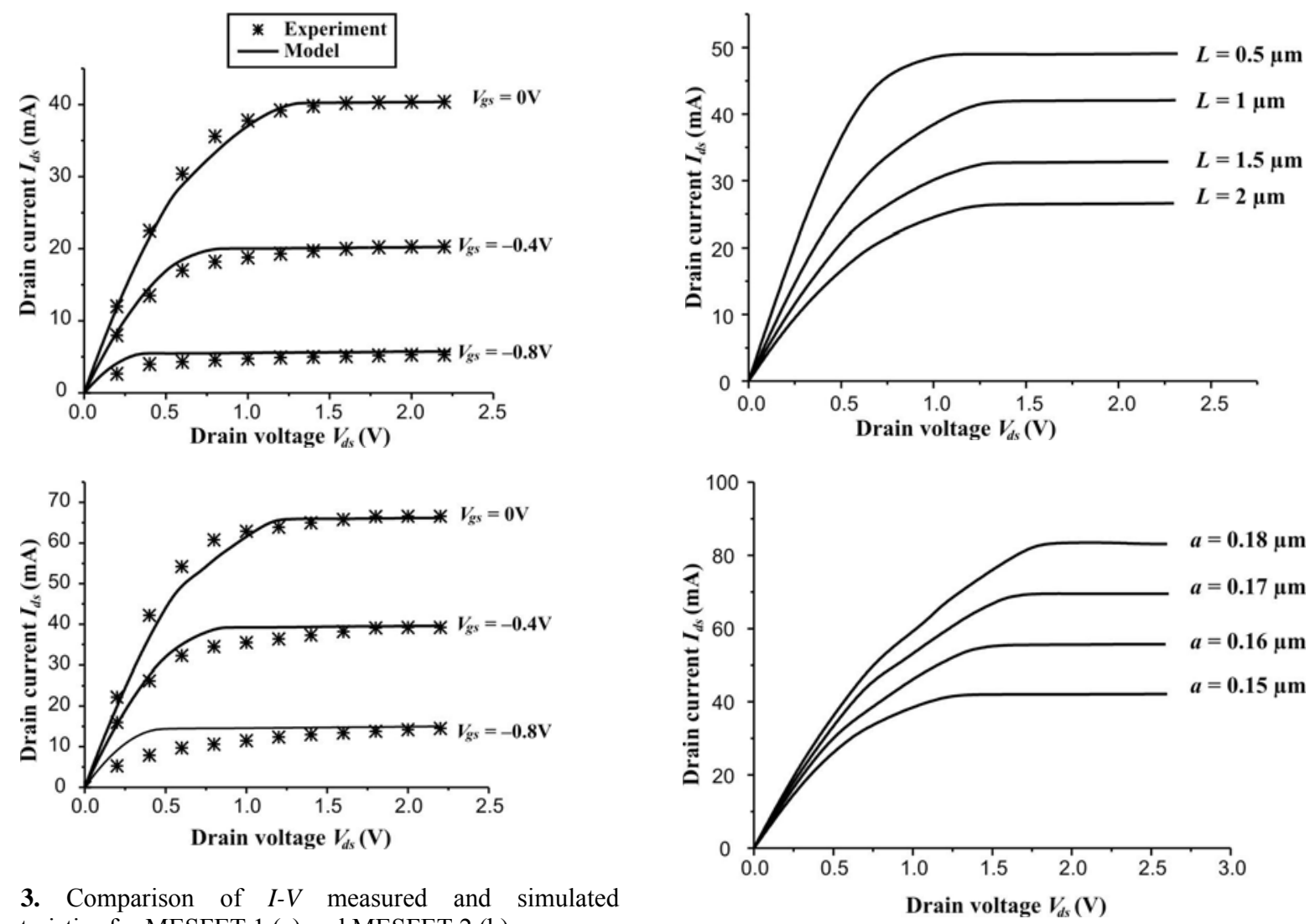

Fig. 3. Comparison of $I-V$ measured and simulated characteristics for MESFET 1 (a) and MESFET 2 (b).
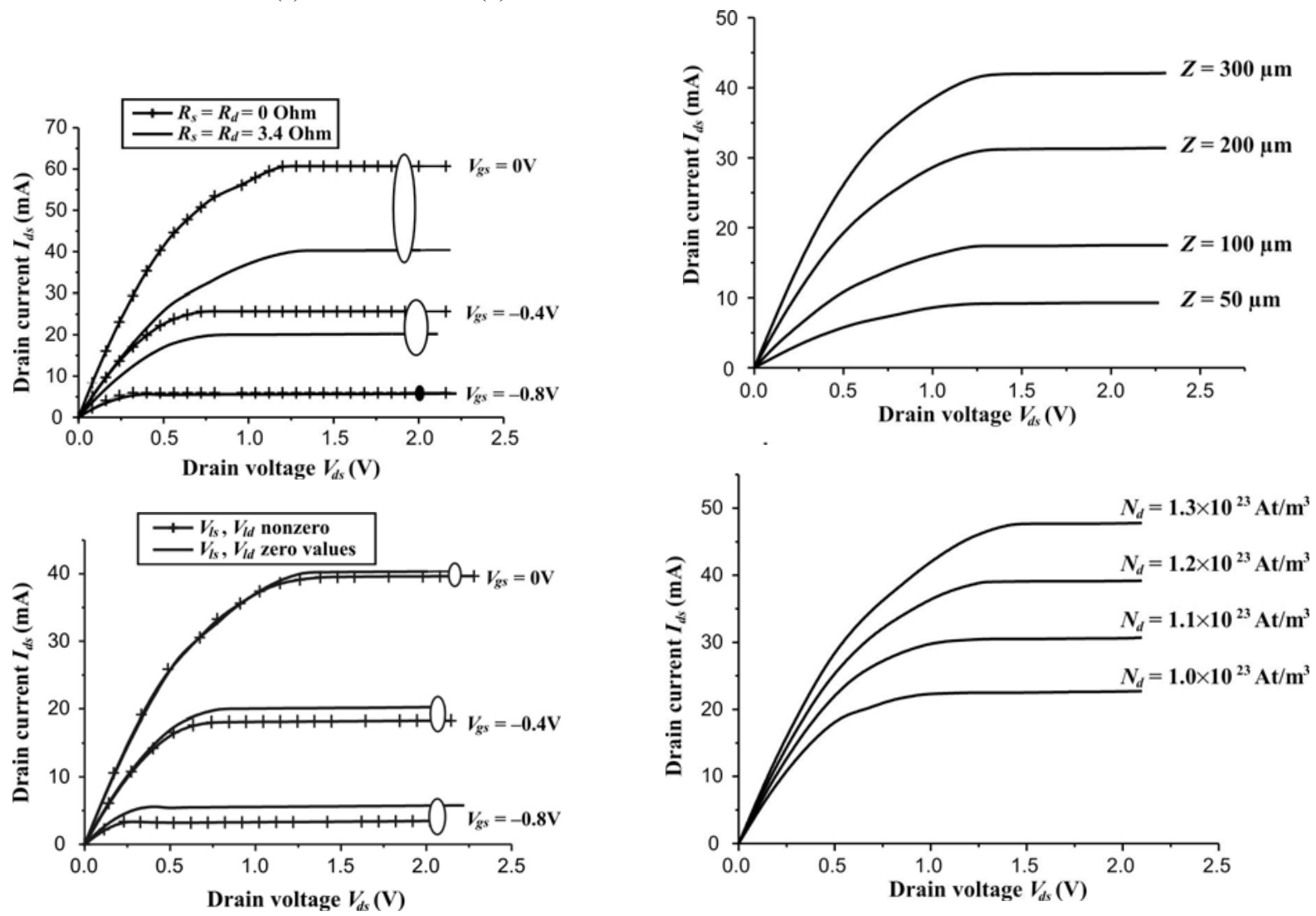

Fig. 4. Effect of the parasite elements on the $I-V$ characteristics: resistances $R_{s}$ and $R_{d}$ (a) and side voltages $V_{l s}$ and $V_{l d}(\mathrm{~b})$.

Fig. 5. Current-voltage characteristic variations for the transistors: MESFET 3 (a), MESFET 4 (b), MESFET 5 (c), MESFET 6 (d).

(C) 2006, V. Lashkaryov Institute of Semiconductor Physics, National Academy of Sciences of Ukraine 


\subsection{Effect of physical and geometrical parameters}

In our numerical simulation $[8,9]$, we have studied the influence of different physical and geometrical parameters $L$ (length of the gate), a (thickness of the active layer), $Z$ (width of the gate) and $N_{d}$ (doping) on the electrical characteristics of the GaAs MESFET transistors, parameters of which are gathered in Table 2.

Figs $5 \mathrm{a}, \mathrm{b}$, c, and $\mathrm{d}$ show the drain current $I_{d s}$ variations as a function of the drain voltage $V_{d s}$ for different physical and geometrical parameters $L, a, Z$, and $N_{d}$.

\section{Table 2.}

\begin{tabular}{|c|c|c|c|c|}
\hline Transistor & $a_{1}$ & $b_{1}$ & $C_{1}$ & $V_{l} / V_{p}$ \\
\hline MESFET 1 & -0.10 & 0.10 & 0.05 & 0.01 \\
\hline MESFET 2 & -0.14 & 0.10 & 0.04 & 0.01 \\
\hline
\end{tabular}

\section{General conclusion}

In this paper, we have proposed an analytical study of the current-voltage characteristics of the GaAs MESFET using the simulation software. The influence of parasite elements, physical and geometrical parameters on these characteristics has been clearly established. The obtained results allow the focusing of the components geometry adapted to specific uses.

\section{References}

1. S.P. Chin, C.Y. We // IEEE Trans. Electron. Devices 40, No 4, p. 712-720 (1993).

2. C.S. Chang, D.Y. Day // IEEE Trans. Electron. Devices 36, No 2, p. 269- 280 (1989).

3. K. Fujii et al. // IEEE Trans. M.T.T. 48, No 3, p. 431-436 (2000).

4. S.P. Murray, K.P. Roenker // Solid State Electronics 46, p. 1495-1505 (2002).

5. T.A. Fjedley, T. Yterdal, M.S. Shur, Introduction to device modeling and circuit simulation. Wiley, New York, 1998.

6. K.M. Shin, D.P. Klamer, J.I. Lion // Solid State Electronics 35, No 11, p. 1639-1640 (1992).

7. C. Leifso et al. // IEEE Trans. Electron. Devices 47, No 5, p. 905-909 (2000).

8. S. Khemissi, Master thesis. Faculty of Sciences. Constantine University, 2003.

9. N. Merabtine, Ph.D thesis. Faculty of Engineering. Constantine University, 2003. 